\title{
Insect cell glycosylation and its impact on the functionality of a recombinant intracrystalline nacre protein, AP24. Supporting Information.
}

Eric P. Chang, ${ }^{1+}$ Iva Perovic, ${ }^{1 \uparrow}$ Ashit Rao, ${ }^{2}$ Helmut Cölfen, ${ }^{2}$ and John Spencer Evans $^{1 *}$

${ }^{1}$ Laboratory for Chemical Physics, Division of Basic Sciences and Center for Skeletal Biology, New York University, 345 E. 24th Street, NY, NY, 10010 USA.

${ }^{2}$ Department of Chemistry, Physical Chemistry, Universität Konstanz, Universitätstrasse 10, Konstanz D78457, Germany.

*To whom correspondence should be addressed: John Spencer Evans, Laboratory for Chemical Physics, Center for Skeletal Biology, New York University College of Dentistry, 345 E. 24th Street, New York, NY, 10010. Tel.: (347) 753-1955 Fax: (212) 995-4087. Email: jse1@nyu.edu

This research was supported by the U.S. Department of Energy, Office of Basic Energy Sciences, Division of Materials Sciences and Engineering under Award DE-FG02-03ER46099. 


\section{TABLE OF CONTENTS}

I. Recombinant expression and analyses of rAP24N and rAP24G.

II. Glycosylation mapping of tag-free purified rAP24G insect cell-expressed variant mixture.

III. Potentiometric $\mathrm{Ca}(\mathrm{II})$ titrations of rAP24N, rAP24G proteins

I. Recombinant expression and analyses of rAP24N and $r A P 24 G$.

\section{rAP24-gp67-H6-TEV (rAP24G)}

gP67-HHHHHHENLYFQ | GDDDEDASSGLCNQYNQNVTTRPNNKPKMFLRKNINFEIISVHNI WRDPNTVYWCDFSLEEEDGIKHWRHYDFNATHWWVEKGCSGTFVVEECNTKDITNPGPR STAGKSPMQGTLAAPKPVANWMSIMSRSRFDMGTWDKEGFNML

\section{rAP24-TrX-H6-TEV (rAP24N)}

TrX-HHHHHHENLYFQ | GDDDEDASSGLCNQYNQNVT TRPNNKPKMFLRKNINFEIISVHNI WRDPNTVYWCDFSLEEEDGIKHWRHYDFNATHWWVEKGCSGTFVVEECNTKDITNPGPR STAGKSPMQGTLAAPKPVANWMSIMSRSRFDMGTWDKEGFNML

Figure S1: rAP24N (bacterial expression, E. coli) and rAP24G (insect cell expression, Sf9) subclone sequences (Trx sequence in green, gp67 envelope glycoprotein secretion signal sequence in red, $\mathrm{His}_{6}$ tag in blue, TEV cleavage site in brown, vertical line denotes site of cleavage) Postcleavage form of the rAP24 protein begins with Gly in the sequence above (i.e., total sequence length of both recombinants are is $147 \mathrm{AA}$, with G1 at the N-terminus and L147 at the C-terminus).

Figure S2: SDS-PAGE analysis (Coomassie Blue staining) of tag-free, purified rAP24N and rAP24G.

For rAP24N, a 12\% Tris-Glycine Novex gel was used. Lane 1: $2 \mu \mathrm{g}$ BSA. Lane $\mathrm{M}_{\mathrm{r}}$ : MW standards (in $\mathrm{kDa}$ ), Lane 2: $3 \mu \mathrm{g}$ rAP24N.

For rAP24G, a 4-20\% gradient gel was used. Lane 1: $2 \mu \mathrm{g}$ BSA. Lane $\mathrm{M}_{\mathrm{r}}$ : $\mathrm{MW}$ standards (in $\mathrm{kDa}$ ). Lane 2: $2 \mu \mathrm{g}$ rAP24G (3 detected variants corresponding to bands $3,2,1$ ).

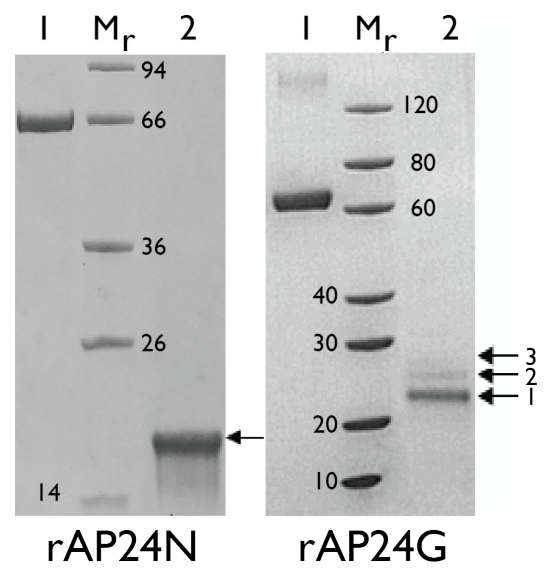



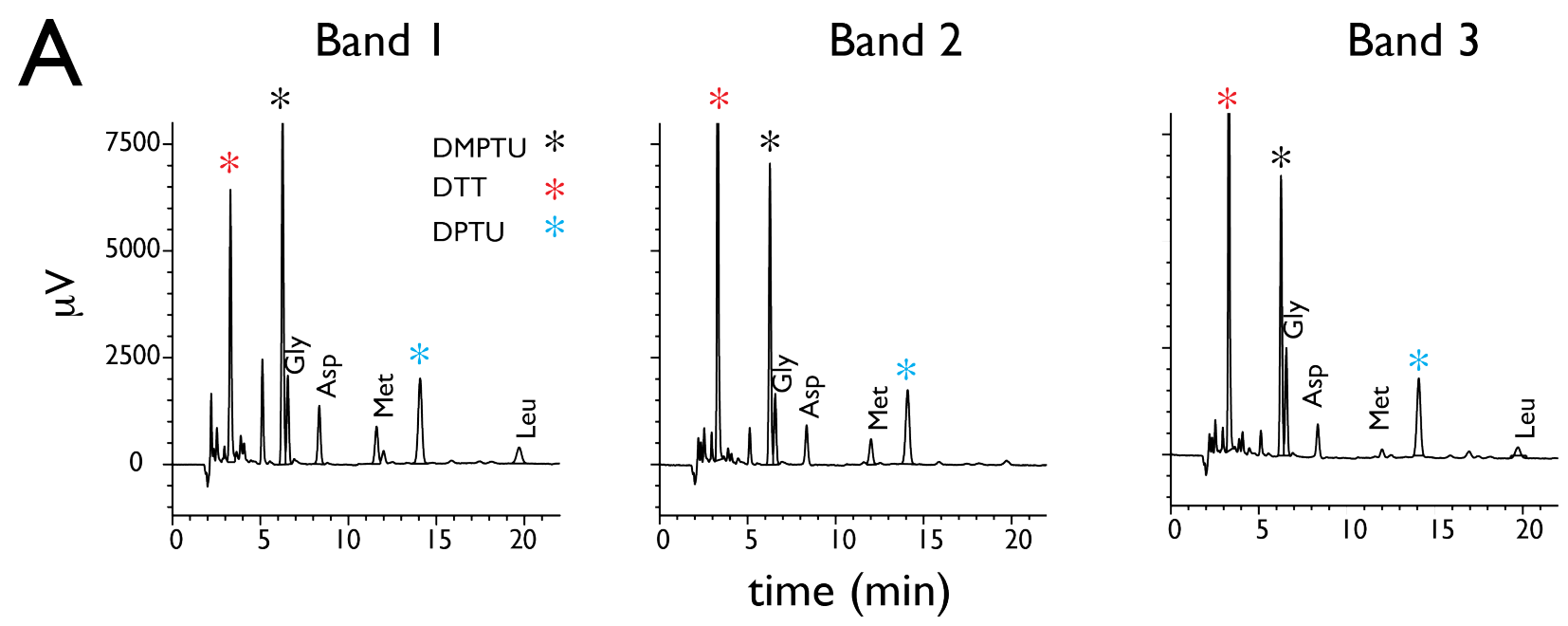

B

\section{Cycle}

$\begin{array}{lcccccccc} & \text { I } & 2 & 3 & 4 & 5 & 6 & 7 & 8 \\ \text { Theoretical } & \text { G } & \text { D } & \text { D } & \text { D } & \text { E } & \text { D } & \text { A } & \text { S } \\ \text { Band I } & \text { G,D,M,L } & \text { D,A,M } & \text { D,E,F,L } & \text { D,E,H } & \text { E,D,S,H } & \text { D,A,H,V } & \text { A,S,H,F } & \text { S,H,A } \\ \text { Band 2 } & \text { G,D,M, } & \text { D,A,E } & \text { D,E,L } & \text { D,E,H,G } & \text { E,D,N,H } & \text { D,A,E,H } & \text { A,S,H,F } & \text { S,S,H } \\ \text { Band 3 } & \text { G,D,M,L } & \text { D,A,V } & \text { D,E,F } & \text { D,E,G } & \text { E,D,I,N } & \text { D,A,H,V } & \text { A,S,H,F } & \text { S,H,Y }\end{array}$

Figure S3. A) Representative N-terminal sequencing first cycle chromatograms of tag-free, purified rAP24G variants (Fig S2, Lane 2). Bands 1,2,3 from Fig S2 were eluted using the protocol described in the manuscript. Subsequently these samples were subjected to Edman degradation using a Shimadzu PPSQ-33A N-terminal sequencer. Eight cycles were performed on each band sample. Parallel runs were conducted using a blank buffer sample and a recombinant human parathyroid hormone (rhPTH) sample for comparison (data not shown). DTT = dithiothreitol; DMPTU = N-dimethyl-N'-phenylthiourea; DPTU = N,N'-diphenylthiourea. B) Theoretical and detected Band 1, 2, 3 N-terminal sequences (8 cycles). Under each cycle are the detected amino acids, with the most significant amino acid listed first. 


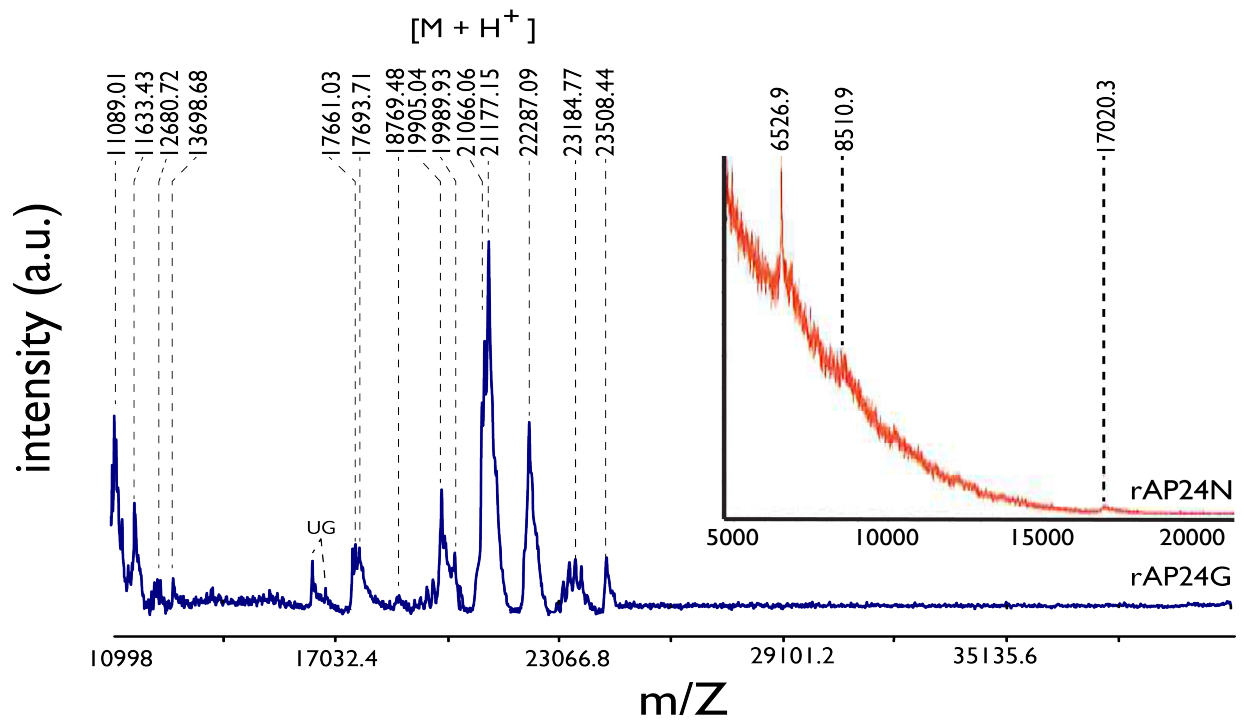

Figure S4. MALDI-TOF-MS spectra (positive ion mode) of tag-free, purified rAP24G and rAP24N (inset spectra). For rAP24G, the adducts from 17661.103 to 23508.44 represent the singly charged species $\left[\mathrm{M}+\mathrm{H}^{+}\right]$and major adducts are annotated on the spectrum. Other adducts at $13698.68 \mathrm{Da}$ and lower mass represent doubly charged adduct species. "UG" refers to minor peaks near $17 \mathrm{kDa}$ that represent unglycosylated rAP24 polypeptides. For rAP24N, the singly (17020.3 Da) and doubly (8510.9 Da) charged adducts were detected along with an unidentified species at $6526.9 \mathrm{Da}$. Note that due to aggregation issues the desorption of rAP24N from the MALDI matrices was very low and hence the signal intensities are weaker and baseline more distorted compared to rAP24G. 
II. Glycosylation mapping of tag-free purified rAP24G insect cell-expressed variant mixture.

Table S1: AP24G MALDI-TOF-MS adducts, monosaccharide matching, and corresponding oligosaccharide structure.

\begin{tabular}{|c|c|c|c|c|c|}
\hline $\mathbf{m} / \mathbf{Z}$ & Adduct & Monosaccharide & $\Delta \mathrm{Da}$ & Oligosaccharide & Possible Structure \\
\hline 720.2 & $\mathrm{~N} / \mathrm{A}$ & No match & & & \\
\hline 836.8 & $\mathrm{M}-\mathrm{H}$ & $\mathrm{NeuAcHexNAc}_{2}$ & 1.14 & Sialylated O-glycan & $\diamond$ \\
\hline 882 & N/A & No match & & & \\
\hline \multirow[t]{2}{*}{949.6} & M-H & $\mathrm{SulfHex}_{2} \mathrm{HexNAc}_{2}$ & 0.85 & Sulfate O-glycan & \\
\hline & & $\mathrm{HexNAc}_{4}$ & -1.19 & O-glycan & \\
\hline \multirow[t]{2}{*}{1030.6} & $\mathrm{M}-\mathrm{H}$ & $\mathrm{Man}_{3} \mathrm{GlcNAc}_{2}$ & -0.22 & $\mathrm{~N}$-glycan core & \\
\hline & & $\mathrm{SulfHexNAc}_{4}$ & -0.25 & Sulfated O-glycan & \\
\hline \multirow[t]{2}{*}{1110.5} & $\mathrm{M}-\mathrm{H}$ & $\mathrm{SulfHex}_{3} \mathrm{HexNAc}_{2}$ & -0.39 & Sulfated O-glycan & \\
\hline & & $\mathrm{Sulf}_{2} \mathrm{HexNAc}_{4}$ & -0.42 & Bisulfated O-glycan & \\
\hline \multirow[t]{2}{*}{1177.1} & M-H & $\operatorname{Man}_{3} \operatorname{GlcNAc}_{2}(\mathrm{Fuc})$ & 0.132 & $\mathrm{~N}$-glycan core plus fucose & \\
\hline & & $\mathrm{NeuGcHex}_{2} \mathrm{HexNAc}_{2}$ & 1.160 & Sialylated O-glycan & \\
\hline
\end{tabular}




\begin{tabular}{|c|c|c|c|c|c|}
\hline $\mathbf{m} / \mathbf{Z}$ & Adduct & Monosaccharide & $\Delta \mathbf{D a}$ & Oligosaccharide & Possible Structure \\
\hline 1272.9 & M-H & $\mathrm{Sulf}_{2} \mathrm{HexHexNAc}_{4}$ & -0.158 & Bisulfated N-glycan & \\
\hline 1286.1 & $\begin{array}{l}\mathrm{M}+\mathrm{Na}- \\
2 \mathrm{H}\end{array}$ & $\mathrm{NeuAcFucHex}_{2} \mathrm{HexNAc}_{2}$ & 1.016 & Sialylated, fucosylated O-glycan & \\
\hline 1354.9 & M-H & $\mathrm{Hex}_{2}+\mathrm{Man}_{3} \mathrm{GlcNAc}_{2}$ & -0.209 & Oligomannose 5 & \\
\hline \multirow[t]{2}{*}{1435.3} & $\mathrm{M}-\mathrm{H}$ & $\mathrm{HexNAc}_{2}+\mathrm{Man}_{3} \mathrm{GlcNAc}_{2}$ & -1.914 & $\begin{array}{l}\text { Agalacto biantennary N-glycan } \\
\text { (G0) }\end{array}$ & \\
\hline & & $\mathrm{NeuAc}_{2} \mathrm{FucHexHexNAc}_{2}$ & 0.101 & $\begin{array}{l}\text { Bisialylated, fucosylated O- } \\
\text { glycan }\end{array}$ & \\
\hline 1488.3 & $\begin{array}{l}\mathrm{M}+\mathrm{Na}- \\
2 \mathrm{H}\end{array}$ & $\mathrm{NeuAcFucHex}_{2} \mathrm{HexNAc}_{3}$ & 0.021 & Sialylated, fucosylated O-glycan & \\
\hline \multirow[t]{2}{*}{1517.6} & M-H & $\mathrm{Hex}_{3}+\mathrm{Man}_{3} \mathrm{GlcNAc}_{2}$ & 0.348 & Oligomannose 6 & \\
\hline & & $\mathrm{SulfHexNAc}_{2}+\mathrm{Man}_{3} \mathrm{GlcNAc}_{2}$ & 0.321 & Sulfated biantennary N-glycan & \\
\hline 1601.0 & M-H & $\mathrm{HexHexNAc}_{2}+\mathrm{Man}_{3} \mathrm{GlcNAc}_{2}$ & 1.642 & $\begin{array}{l}\text { Monogalacto biantennary N- } \\
\text { glycan (G1) }\end{array}$ & \\
\hline
\end{tabular}




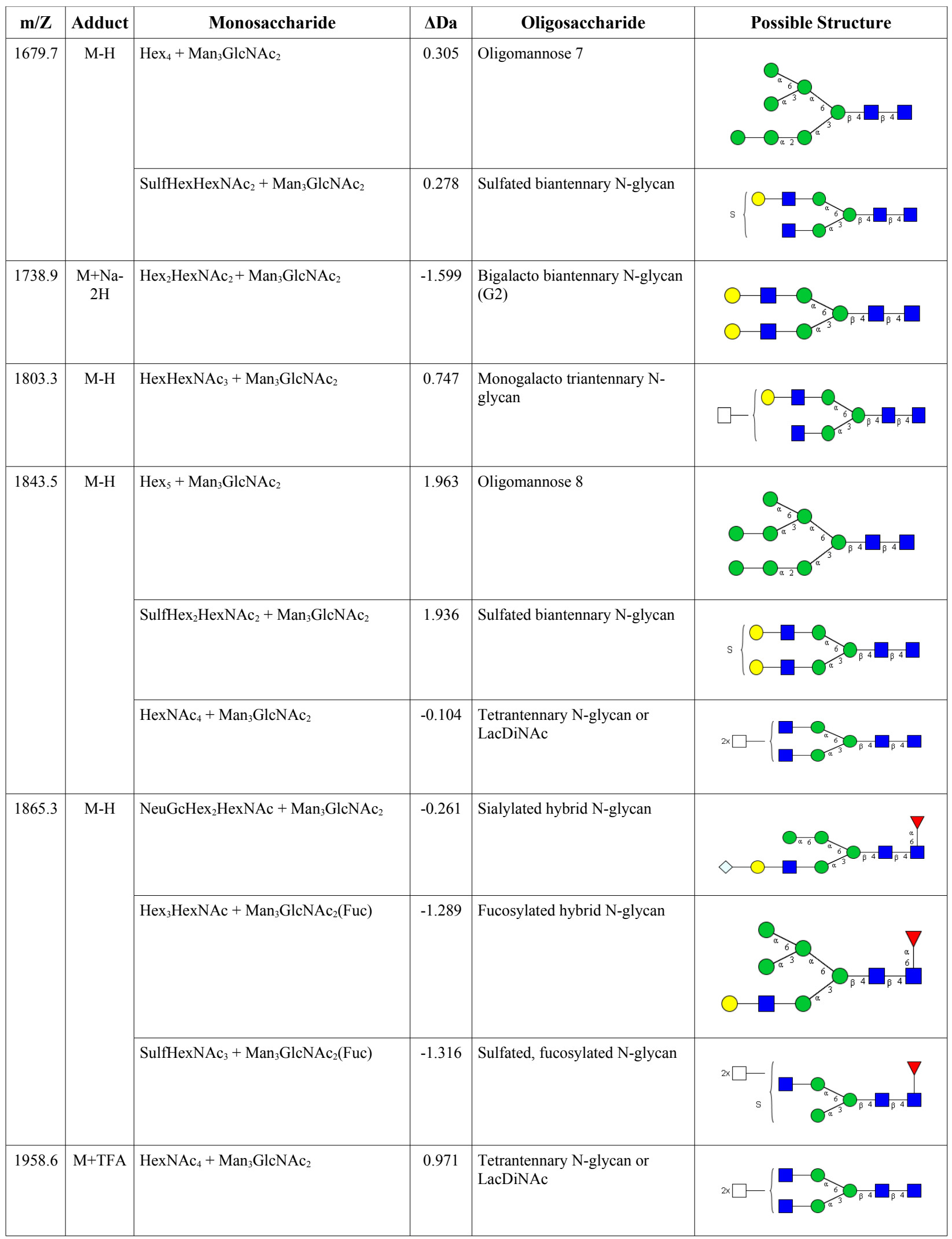




\begin{tabular}{|c|c|c|c|c|c|}
\hline $\mathbf{m} / \mathbf{Z}$ & Adduct & Monosaccharide & $\Delta \mathbf{D a}$ & Oligosaccharide & Possible Structure \\
\hline \multirow[t]{3}{*}{2004.4} & \multirow[t]{3}{*}{$\mathrm{M}-\mathrm{H}$} & $\mathrm{Hex}_{6}+\mathrm{Man}_{3} \mathrm{GlcNAc}_{2}$ & 0.720 & Oligomannose 9 & \\
\hline & & $\mathrm{Sulf}_{2} \mathrm{HexNAc}_{4}+\mathrm{Man}_{3} \mathrm{GlcNAc}_{2}$ & 0.666 & Bisulfated N-glycan & \\
\hline & & $\mathrm{HexHexNAc}_{4}+\mathrm{Man}_{3} \mathrm{GlcNAc}_{2}$ & -1.347 & $\begin{array}{l}\text { Monogalacto tetrantennary N- } \\
\text { glycan }\end{array}$ & \\
\hline \multirow[t]{2}{*}{2135.3} & \multirow[t]{2}{*}{ M-H } & $\mathrm{SulfFuc}_{2} \mathrm{Hex}_{2} \mathrm{HexNAc}_{2}+\mathrm{Man}_{3} \mathrm{GlcNAc}_{2}$ & 1.450 & $\begin{array}{l}\text { Sulfated, bifucosylated N- } \\
\text { glycan }\end{array}$ & \\
\hline & & $\mathrm{NeuAcHexNAc}_{4}+\mathrm{Man}_{3} \mathrm{GlcNAc}_{2}$ & 0.437 & Monosialo N-glycan & \\
\hline \multirow[t]{2}{*}{2166.1} & \multirow[t]{2}{*}{$\mathrm{M}-\mathrm{H}$} & $\mathrm{SulfHexHexNAc}_{4}+\mathrm{Man}_{3} \mathrm{GlcNAc}_{2}$ & 0.224 & Sulfated, monogalacto N-glycan & \\
\hline & & $\mathrm{Hex}_{2} \mathrm{HexNAc}_{4}+\mathrm{Man}_{3} \mathrm{GlcNAc}_{2}$ & -1.789 & Bigalacto N-glycan & \\
\hline
\end{tabular}

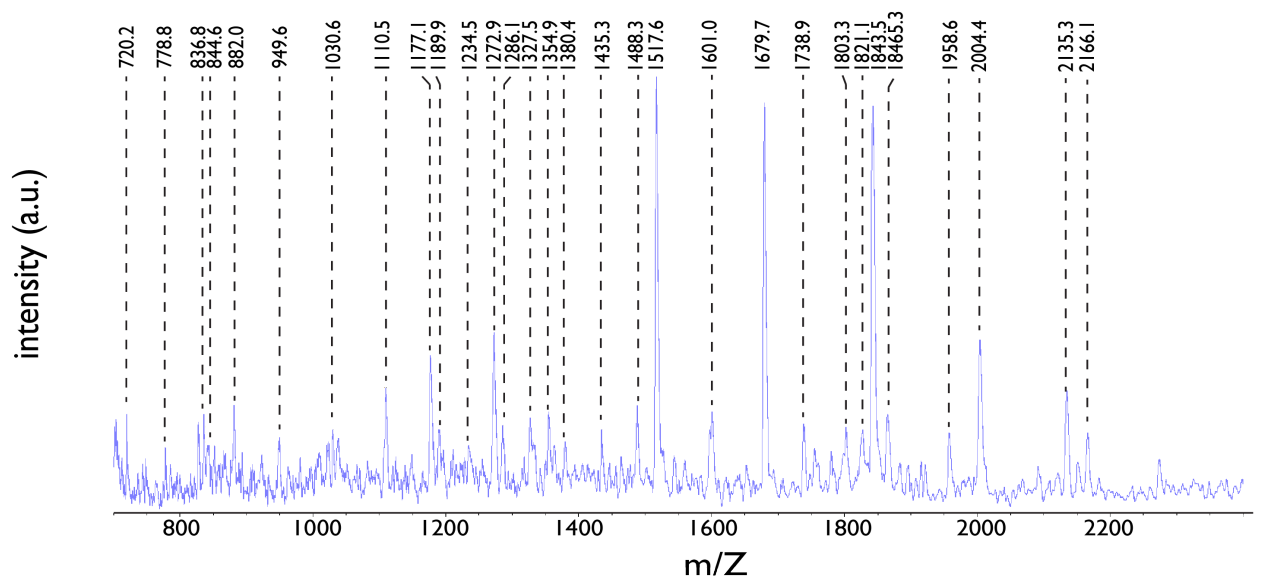

Figure S5: MALDI-TOF-MS spectra (negative ion mode) of tag-free purified rAP24G N- and O-linked oligosaccharide profile. The $\mathrm{m} / \mathrm{Z}$ values for annotated adduct species are listed in Table $\mathrm{S} 1$. Note that the assignments in Table S1 are tentative and may be affected by the weak intensities of the adduct species. 
III. Potentiometric $\mathrm{Ca}(\mathrm{II})$ titrations of $\mathrm{rAP} 24 \mathrm{~N}, \mathrm{rAP} 24 \mathrm{G}$ proteins

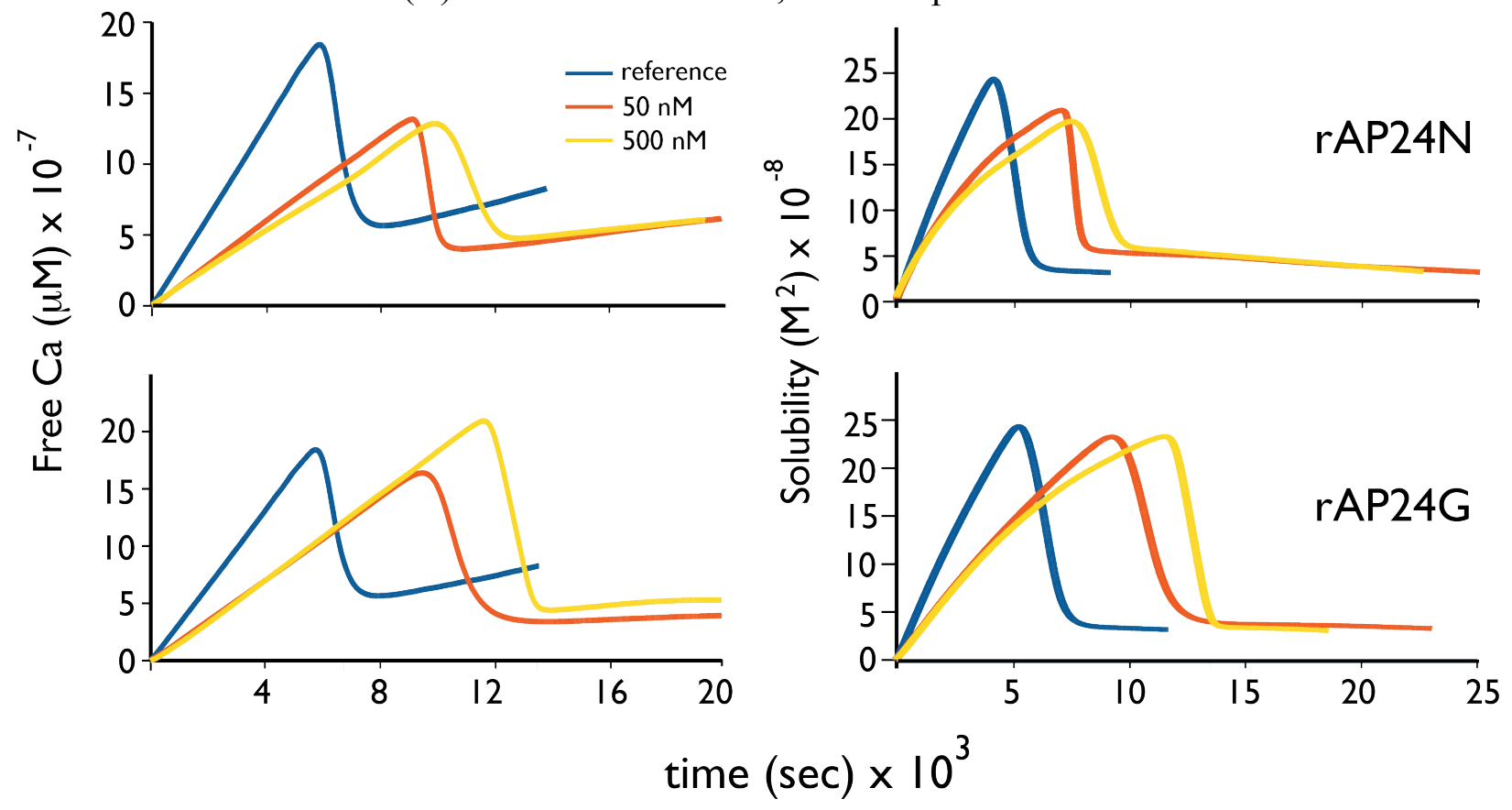

Figure S6: Development of free $\mathrm{Ca}$ (II) ion concentration (LEFT) and calcium carbonate solubility product (RIGHT) in potentiometric titrations of 50 and $500 \mathrm{nM} \mathrm{rAP} 24 \mathrm{~N}$ (TOP) and rAP24G (BOTTOM) proteins in $10 \mathrm{mM}$ carbonate buffer, $\mathrm{pH} 9.0$ as a function of time. In each plot the reference curve refers to parallel experiments conducted in the absence of protein.

The standard potentiometric curve provides information on the formation and stabilities of PNCs in solution. As free $\mathrm{Ca}^{2+}$ is added to the carbonate solution, ion complexes (i.e., PNCs) form and this is represented by the initial linear region of the titration curve. Where the measured free $\mathrm{Ca}^{2+}$ decreases upon further addition of $\mathrm{CaCl}_{2}$ (i.e., the peak region), this marks the start of solid phase nucleation (e.g., ACC) from PNCs. With respect to PNC stability, the slope of the prenucleation regime (i.e., the initial linear region) provides indirect evidence of the interaction between additive molecules and solute ion associates, leading to PNC stabilization (i.e., Slope Additive $_{<}$ Slope $_{\text {Ref }}$ ) or destabilization (Slope Additive $>$ Slope $_{\text {Ref }}$ ). 